AperTO - Archivio Istituzionale Open Access dell'Università di Torino

\title{
European Myeloma Network: the 3rd Trialist Forum Consensus Statement from the European experts meeting on multiple myeloma.
}

\section{This is the author's manuscript}

Original Citation:

\section{Availability:}

This version is available http://hdl.handle.net/2318/80282

since

Terms of use:

Open Access

Anyone can freely access the full text of works made available as "Open Access". Works made available under a Creative Commons license can be used according to the terms and conditions of said license. Use of all other works requires consent of the right holder (author or publisher) if not exempted from copyright protection by the applicable law. 


\section{(3) \\ UNIVERSITÀ DEGLI STUDI DI TORINO}

This is an author version of the contribution published on:

Questa è la versione dell'autore dell'opera:

[Leuk Lymphoma. 2010 Nov;51(11):2006-11. doi: 10.3109/10428194.2010.516378. Epub 2010 Aug 31]

The definitive version is available at:

La versione definitiva è disponibile alla URL:

[http://informahealthcare.com/doi/abs/10.3109/10428194.2010.516378] 


\title{
European Myeloma Network: the 3rd Trialist Forum Consensus Statement from the European experts meeting on multiple myeloma
}

Monika Engelhardt, Josefina Udi, Martina Kleber, Andrew Spencer, Alberto Rocci, Stefan Knop, Benedetto Bruno, Sara Bringhen, José A. Pérez-Simón, Sonja Zweegman, Christoph Driessen, Francesca Patriarca, Martin Gramatzki, Evangelos Terpos, Orhan Sezer, Martin Kropff, Christian Straka, Hans E. Johnsen, Anders Waage, Martin Boegsted, Henk Lokhorst, Roman Hájek, Gareth Morgan, Mario Boccadoro, Heinz Ludwig, Michele Cavo, Aaron Polliack, Pieter Sonneveld, Hermann Einsele, Antonio Palumbo

\begin{abstract}
Over the past two decades, not only treatment options, but also the diagnosis, staging, and risk assessment of multiple myeloma (MM), have undergone significant development, partially due to a deeper understanding of MM pathogenesis. Conventional cytogenetics and fluorescence in situ hybridization are routinely assessed in MM, and when combined with ISS stage may attain an even better predictive potential. In order to achieve even more effective and individualized therapies, one crucial goal is the identification of genes and gene combinations that predict for response or resistance to chemotherapy. High-dose chemotherapy with autologous stem cell transplant (SCT) still remains the standard therapy for younger patients, with novel agents now being included in both pre-transplant regimens and post-transplant consolidation/maintenance approaches. Similarly, novel agents are also being incorporated into allogeneic SCT for selected patients. In the treatment of elderly patients with MM, novel agents have been successfully incorporated into less intensive regimens, including melphalan/prednisone, low-dose dexamethasone, and cyclophosphamide/dexamethasone. While second-generation proteasome inhibitors are currently being intensively investigated, the subcutaneous administration of bortezomib, being equivalent to the established i.v. route, is now entering clinical practice. Supportive care remains a crucial aspect in the management of MM. The European Myeloma Network Trialist Group aims to address these contemporary aspects in MM.
\end{abstract}

\section{Introduction to the European Myeloma Network Trialist Group and the 3rd Trialist Forum}

The European Myeloma Network (EMN), founded in 2005, represents an international cooperative network, including members of the GIMEMA (Gruppo Italiano Malattie Ematologiche dell'Adulto), HOVON (Hemato Oncology Foundation for Adults in The Netherlands), DSMM (Deutsche Studiengruppe Multiples Myelom), and other international study groups, for the diagnosis and treatment of multiple myeloma (MM). As in previous years, an international meeting of the EMN Trialist Group (TG) was held in Baveno, Italy, on 16-17 May 2010. This article summarizes the activities of the EMN-TG and the 3rd Trialist Forum, with most of the relevant aspects discussed at this meeting. 


\section{Phase III protocols for younger patients with multiple myeloma}

\section{Michele Cavo, Pieter Sonneveld, Benedetto Bruno}

High-dose therapy with autologous stem cell transplant (ASCT) is still considered the standard treatment approach for younger patients with MM (18-65 years, as well as up to 75 years with good performance status in clinical trials). The impact of different pre-transplant regimens including novel agents on stem cell mobilization, response, and survival in patients undergoing ASCT is being intensively investigated $[1,2]$. Results from phase II/III studies including novel agents in the induction treatment have shown an increase in complete remission/very good partial remission (CR/VGPR) rates not only pre- but also post-ASCT. However, non-transplant approaches with novel agent-based induction and ASCT performed at the time of relapse are currently also being evaluated. Questions that still remain to be answered are the role of ASCT versus novel agents, tandem versus single ASCT, and novel agent maintenance versus non-maintenance approaches. One innovative GIMEMA protocol presented at the meeting consists of induction therapy with three cycles of bortezomib, cyclophosphamide, and dexamethasone (VCD) followed by cyclophosphamide-induced stem cell mobilization and first randomization to either four cycles of bortezomib, melphalan, and prednisone (VMP) or MEL200 (melphalan $200 \mathrm{mg} / \mathrm{m}^{2}$ ) followed by single or tandem ASCT, with plans to present the preliminary findings at the American Society of Hematology (ASH) Meeting later this year [3]. After VMP or MEL200, patients will undergo a second randomization to receive consolidation with bortezomib, lenalidomide, and dexamethasone (VRD) versus no consolidation. The presence of cytogenetic abnormalities, such as deletion of chromosome 1p [del(1p)], amplification of chromosome 1q [ampl(1q)], translocation of chromosomes 4 and 14 [t(4;14)], 14 and 16 [t(14;16)], and 11 and 14 [t(11;14)], amplification of chromosome 9 , and the deletion of chromosome $17 \mathrm{p}[\operatorname{del}(17 \mathrm{p})]$ as detected by fluorescence in situ hybridization (FISH) and deletion of chromosome 13 by conventional cytogenetics (CG) are assessed at diagnosis. Furthermore, immunophenotyping at diagnosis, during treatment, and at follow-up to enable the confirmation of a stringent complete remission ( $\mathrm{SCR}$ ) is incorporated in the protocol, and translational studies such as gene expression profiling (GEP) on purified MM plasma cells (PCs) and single nucleotide polymorphism (SNP) analysis for loss of heterozygosity (LOH) are also planned to potentially inform individualized treatment approaches in future trials.

\section{Phase II salvage protocols}

\section{Jose A. Perez-Simon, Henk Lokhorst, Benedetto Bruno}

Allogeneic stem cell transplant (allo-SCT) appears to be a potentially curative strategy in MM, and is currently pursued worldwide within clinical trials. Novel agents, such as bortezomib, thalidomide, and lenalidomide, are being incorporated into induction regimens prior to allo-SCT in an attempt to increase the extent of cytoreduction before SCT, as well as in post-transplant consolidation or maintenance regimens to enhance anti-MM and graft-versus-myeloma effects. Since last year's EMN-TG meeting at Stresa, prospective transplant protocols have been designed to further evaluate the role of new drugs as induction before allo-SCT, and their toxicity, tolerability, and efficacy for maintenance when used as treatment after allo-SCT [4].

The phase II HOVON 76 study has examined the efficacy and safety of maintenance using $10 \mathrm{mg}$ lenalidomide for 21 days each month, starting between 1 and 6 months after non-myeloablative (RIC) allo-SCT. Common toxicities were hematologic side effects and exacerbation of acute graftversus-host disease (GvHD) that only $58 \%$ of patients could continue lenalidomide beyond the fourth cycle [5]. Thus, future allo-SCT strategies may include increased conditioning intensity regimens within RIC protocols, improvement in GvHD and relapse prevention strategies, and 
combination approaches to capture both, e.g. combined rapamycin and bortezomib use, for both GvHD prophylaxis and MM maintenance treatment.

\section{EMN phase II melphalan chemoprediction trial}

\section{Hans E. Johnsen, Martin Boegsted}

The main cause of treatment failure in malignant diseases, and in particular in MM, is the acquisition of resistance to anti-cancer agents in tumor cells. Understanding the mechanisms leading to multidrug resistance (MDR) is important for the development of even more effective and individualized therapies in MM. Moreover, the identification of genes or gene combinations responsible for drug resistance may be helpful to predict response versus resistance to chemotherapy in patients with MM.

The working hypothesis of this EMN translational study has been that the combination of drug screens of B-lineage cancer cell lines and gene expression profiles (GEPs) can establish a genetic model for predicting drug resistance in patients with MM, which can be clinically validated in newly diagnosed candidates for high-dose melphalan and ASCT. The specific aims of the study are (1) to compare drug screens for melphalan of the NCI60 and B-cell lines, (2) to use a multivariate regression technique to develop a melphalan treatment predictor, and (3) to validate this predictor retrospectively in available clinical data sets from Arkansas Cancer Center ASCT-treated patients receiving 'total therapy.' To date the present study has established a melphalan resistance index based on genes involved in cancer and MM pathogenesis that may predict outcome following highdose melphalan. The impact of the generated gene list will be prospectively validated and correlated to known biomarkers in independent data sets, as planned in an upcoming prospective EMN intergroup trial.

\section{Phase III protocols for elderly patients with multiple myeloma}

\section{Orhan Sezer, Sara Bringhen, Christian Straka}

Reduced high-dose melphalan $\left(100-140 \mathrm{mg} / \mathrm{m}^{2}\right)$ and ASCT is an option for elderly patients up to the age of 75 years. Patients not eligible for ASCT have historically been treated with the combination of melphalan and prednisone (MP) alone, and more recently in combination with novel drugs. Current trials have shown that the combination of chemotherapy and novel agents significantly improves therapeutic efficacy, with additive or synergistic activity in vivo. Therefore, several studies have investigated the addition of novel agents to the traditional MP regimen. Lenalidomide has been evaluated in combination with MP, with low-dose dexamethasone (Rd), and with cyclophosphamide/prednisone in untreated symptomatic patients with MM. For symptomatic patients aged 65-75 years, Rd with or without intensification by high-dose melphalan followed by ASCT may also be an effective therapeutic option. Dose-reduced tandem-ASCT (MEL140) with a novel induction (Rd) is therefore being tested against Rd without ASCT (e.g. DSMM XIII trial), and results are awaited with anticipation. Current trials also aim to decipher the role of melphalan versus cyclophosphamide, testing their ideal doses in combination with novel agents compared to alkylating-free novel drug combinations (e.g. Rd) in elderly patients. 


\section{Phase II induction protocols}

\section{Antonio Palumbo}

Subcutaneous (s.c.) administration of bortezomib seems equivalent to the established intravenous (i.v.) route, and may represent a convenient alternative for newly diagnosed elderly patients with MM. Subcutaneous bortezomib is also currently being tested in different combinations such as melphalan and prednisone (MP), cyclophosphamide and prednisone, and prednisone alone, followed by maintenance s.c. bortezomib until disease progression. Recent studies have also focused on the development of novel proteasome inhibitors such as NPI-0052, which induces apoptosis in MM cell lines and primary cells from patients with MM in vitro, has been shown to inhibit tumor cell growth in vivo, and is orally bioactive.

\section{Proposal for a substudy on the efficacy of intravenous ferric carboxymaltose in patients with functional iron deficiency}

\section{Heinz Ludwig}

Anemia, defined as a hemoglobin level $<12 \mathrm{~g} / \mathrm{dL}$ (typically appearing as normochromicnormocytic), is a frequent complication (50-70\%) in patients with MM, and, as in other malignant diseases, can arise years before the diagnosis of MM [6]. In cancer patients in general and in particular in patients with MM, a low transferrin saturation (TSAT $<20 \%$ ) is a specific indicator of functional iron deficiency. At this meeting, the panel of experts emphasized the importance of determining (1) the prevalence of iron deficiency (ID) and (2) the efficacy of i.v. iron supplementation to correct ID in MM. The prevalence of ID, including absolute ID (AID) and functional ID (FID), has been investigated in a single center study including 1989 patients with various hematological and malignant diseases. Within the subgroup of patients with MM, ID and ID with anemia occurred in $44 \%$ and $21 \%$ of patients, respectively. Additionally, ID with and without anemia significantly increased with age, and was more frequent in patients older than 80 years than in those $\leq 60$ years [7].

Recently, published data on the treatment of patients with chronic heart failure and ID, with or without anemia, with i.v. ferric carboxymaltose (FCM) suggest that currently available i.v. iron agents are effective in the management of ID and anemia, improving symptoms, functional capacity, and quality of life, while having an acceptable side-effect profile [8]. FCM might therefore be capable of correcting iron deficiency also in patients with $\mathrm{MM}$, and should therefore be of interest to pursue further.

\section{Phase I network and protocols}

\section{Martin Gramatzki}

Important considerations for performing phase I/II trials and their successful completion are welldefined biological rationales to be tested in these studies. Well-designed treatment schedules and precise timing of these studies are important, amongst other aspects. Essential elements for the creation of a phase I network at each center include Good Clinical Practice (GCP)-trained personnel, adequate GCP-clinical trial facilities, intensive care access, basic interest in translational 
research, and sufficient numbers of patients to include in these studies. As these prerequisites are met by the EMN study group, the importance of organizing and implementing a phase I network with the creation of a platform for interested groups and the definition of a subcommittee for this purpose is being planned.

\title{
Cytogenetics and biobanking for basic research: cytogenetics and biobanking protocols
}

\begin{abstract}
Alberto Rocci
Conventional cytogenetics and FISH are routinely assessed prognostic markers in MM. A recently presented large multicenter analysis from the International Myeloma Working Group (IMWG) in 9897 patients with MM evaluated the impact of CG and FISH, when combined with the International Staging System (ISS), on progression-free and overall survival (PFS and OS). The analysis revealed that $\mathrm{t}(4 ; 14)$ most prominently correlated with diminished PFS. The combination of $\mathrm{t}(4 ; 14)$ and ISS stage displayed a significantly increased predictive potential. For ISS stage I without $\mathrm{t}(4 ; 14)$ as opposed to ISS stage III with concomitant presence of $\mathrm{t}(4 ; 14)$, OS rates differed markedly at 4 years $(81 \%$ and $22 \%$, respectively). ISS stage I with $t(11 ; 14)$ revealed the best outcome, with an OS at 4 years of $89 \%$ [9].

Well-organized biobanking is therefore essential for basic and translational research. Samples such as whole bone marrow biopsies, purified CD138+ plasma cells, and serum from all patients enrolled in clinical trials should be collected at diagnosis and stored in reference laboratories. As proliferating cells are needed for conventional cytogenetic studies and these are not frequently obtained, FISH on purified PCs remains the 'gold standard' cytogenetic examination for MM. It appears that it is not essential to perform FISH analysis repeatedly (e.g. at relapse), unless this issue is being addressed in clinical trials, as FISH data have shown less variability with disease progression than initially thought. The recommended essential FISH panel should include $\mathrm{t}(4 ; 14)$, $t(14 ; 16)$, and del(17p13) and optionally $t(11 ; 14)$, 1q gain, and $1 p$ loss, while for conventional cytogenetics del(13q14) and aneuploidy can be determined.
\end{abstract}

\section{Future directions for basic research: common basic research projects}

\section{Gareth Morgan}

The Dutch-German HOVON-65/GMMG (German-speaking Myeloma-Multicenter Group)-HD4 trial has addressed the issue of whether genetic variation is associated with therapeutic outcome [10]. For this purpose, a molecular inversion probe (MIP) SNP chip was used, containing SNPs in genes responsible for cellular functions and pathways that may influence disease response, toxicities, complications, and survival. In this trial, patients were treated with three cycles of either vincristine, dexamethasone, and doxorubicin (VAD) or bortezomib, dexamethasone, and doxorubicin (PAD). Both treatment arms were compared in terms of response and possible SNP association. This analysis identified genetic polymorphisms uniquely associated with VAD or PAD treatment response. The establishment of central biobanks containing serum or plasma samples of homogeneously treated patients with MM is an important ongoing goal of clinical studies. 


\section{MPT meta-analysis}

\section{Anders Waage}

Several MPT trials have studied melphalan and prednisone (MP) versus the combination of MP and thalidomide (MPT) in transplant-ineligible elderly patients with MM. A meta-analysis integrating the existing outcome data related to the efficacy of MP versus MPT was also presented at the ASH 2009 Annual Meeting [11]. Data of six currently available randomized controlled trials (RCTs) have all shown a significant improvement in overall response rate (ORR) with MPT. However, while an improvement in PFS was found in all of them, a significant improvement in OS has not been unequivocally observed. These six current trials comparing MP with MPT comprise a total of 1685 patients, and have shown heterogeneity in terms of drug dosages administered, the number of cycles given, thalidomide maintenance versus no maintenance, and differences in patient selection. Despite these differences, the homogeneity seemed acceptable enough to perform a meaningful meta-analysis. For PFS and OS, data were extractable from four of the trials. MPT induced superior response rates compared with MP, and pooled hazard ratios for PFS and OS were 0.67 (0.55-0.8 median 20.4 vs. 14.9 months; $p=0.001)$ and $0.82(0.66-1.02 ; 39.3$ vs. 32 months; $p=0.085)$, respectively. Using the meta-analysis approach, MPT is confirmed to be superior to MP in terms of response and PFS, whereas an increased, but not significant, OS benefit is obtained.

\section{Conclusions}

Advances in the understanding of the pathogenesis of MM have led to the development of innovative targeted therapies, including the use of histone deacetylase inhibitors (HDAC inhibitors), heat-shock protein-90 (hsp-90) inhibitors, and second-generation proteasome inhibitors, such as carfilzomib [12] and NPI-0052, that can potentially be used either alone or in combination. When determining the most appropriate treatment doses and schedules for patients with MM, age and the presence of co-morbidities (cardiopulmonary disease, renal failure, polyneuropathy, diabetes mellitus, and possibly various other as yet undetermined organ function risks) all need to be considered. Additionally, age-adjusted therapy, and prevention and management of treatment- and MM-related adverse events, including supportive care measures, all need to be taken into account. Improvement in international cooperation, including the formation of the EMN-TG, represents a major achievement, and should lead to more substantial advances in the future management of MM. The ability to perform meta-analyses, such as that of the six randomized controlled trials comparing MP and MPT, which clearly show an improvement in response and PFS with MPT, combined with the fact that larger patient cohorts for meaningful clinical studies and translational research projects are being established, illustrate the need for future international efforts and consortiums which hopefully will contribute to further therapeutic gains for patients with MM. The next EMN-TG meeting has been scheduled for 15-16 May 2011.

Declaration of interest: The authors report no conflicts of interest. The authors alone are responsible for the content and writing of the article.

\section{References}

1. Einsele $\mathrm{H}$, Liebisch $\mathrm{P}$, Langer $\mathrm{C}$, et al Velcade, intravenous cyclophosphamide and dexamethasone (VCD) induction for previously untreated multiple myeloma (German DSMM XIa Trial). Blood 2009;114(Suppl. 1): Abstract 131. 
2. Eleutherakis-Papaiakovou E, Christoulas D, Migkou M, et al The impact of different pretransplant regimens including novel agents on peripheral blood mobilization, harvest, response and survival in patients undergoing ASCT for multiple myeloma. A single-center experience in 210 patients. Blood 2009;114(Suppl. 1): Abstract 2279.

3. Palumbo A, Cavallo F, Yehuda D-B, et al A prospective, randomized study of melphalan, prednisone, lenalidomide (MPR) versus melphalan $(200 \mathrm{mg} / \mathrm{m} 2)$ and autologous transplantation (Mel200) in newly diagnosed myeloma patients: an interim analysis. Blood 2009;114(Suppl. 1): Abstract 350.

4. Engelhardt M, Kleber M, Udi J, et al Consensus statement from European experts on the diagnosis, management, and treatment of multiple myeloma: from standard therapy to novel approaches. Leuk Lymphoma 2010;51:1424-1443.

5. Minnema M-C, van der Holt B, Kersten M-J, et al First interim analysis of HOVON 76: lenalidomide maintenance following non myeloablative allogeneic stem cell transplantation in patients with multiple myeloma. Blood 2009; 114(Suppl. 1): Abstract 2285.

6. Edgren G, Bagnardi V, Bellocco R, et al Pattern of declining hemoglobin concentration before cancer diagnosis. Int J Cancer 2010;127:1429-1436.

7. Ludwig H, Endler G, Hübl W, Klement B, Cushway T. High prevalence of iron deficiency in patients with various hematological and malignant diseases: a single center study in 1989 sequential patients. Haematologica 2010;95(Suppl. 2): Abstract 1819.

8. Anker SD, Comin Colet J, Filippatos G, et al Ferric carboxymaltose in patients with heart failure and iron deficiency. N Engl J Med 2009;361:2436-2448.

9. Avet-Loiseau H, Durie B, Haessler J, et al Impact of FISH and cytogenetics on overall and event free survival in myeloma: an IMWG analysis of 9,897 patients. Blood 2009;114(Suppl. 1): Abstract 743.

10. Corthals S-L, Goldschmidt H, Johnson D-C, et al Genetic associations with therapy response in the HOVON-65/GMMG-HD4 trial in patients with multiple myeloma. Blood 2009;114(Suppl. 1): Abstract 1790.

11. Kapoor P, Rajkumar S-V, Dispenzieri A, et al Melphalan and prednisone (MP) versus melphalan, prednisone and thalidomide (MPT) as initial therapy for previously untreated elderly and/or transplant ineligible patients with multiple myeloma: a meta-analysis of randomized controlled trials. Blood 2009;114(Suppl. 1): Abstract 615.

12. Vij R, Siegel D-S, Kaufman J-L, et al Results of an ongoing open-label, phase II study of cerfilzomib in patients with relapsed and/or refractory multiple myeloma. J Clin Oncol 2010;28(Suppl.): Abstract 8000. 